\title{
UTILIZAÇÃO DE CASCA DE ARROZ E ESTERCO BOVINO COMO SUBSTRATO PARA A MULTIPLICAÇÃO DE Eisenia fetida Savigny (1826)
}

\author{
Rice husk and cattle manure used as substrate for the \\ Eisenia fetida Savigny (1826) multiplication
}

\author{
Zaida Inês Antoniolli ${ }^{1}$, Gerusa Pauli Kist Steffen², Ricardo Bemfica Steffen ${ }^{3}$
}

\begin{abstract}
RESUMO
A utilização de minhocas, principalmente para a alimentação animal, vem sendo adotada e estudada, pois esses organismos apresentam em sua constituição vitaminas e aminoácidos. Entre os materiais possíveis de serem utilizados na minhocultura, atualmente incentiva-se o aproveitamento de resíduos agroindustriais de alta disponibilidade, como a casca de arroz no Rio Grande do Sul. Objetivou-se, neste trabalho, avaliar a eficiência de substratos à base de casca de arroz e esterco bovino na multiplicação e produção de biomassa de minhocas (Eisenia fetida). A população de minhocas foi avaliada quanto ao número de cocons, ao índice de multiplicação (população final/população inicial) e à biomassa de minhocas frescas e secas. A avaliação foi realizada aos 60 dias após a instalação do experimento, com quatro repetições. A análise estatística dos resultados mostrou que a inclusão de casca de arroz favoreceu o desenvolvimento e a taxa de reprodução das minhocas. A maior biomassa de minhocas foi obtida nos tratamentos: $50 \%$ casca de arroz inteira e 50\% esterco bovino, esterco bovino mais casca de arroz inteira tratada com hidróxido de amônio a 20 e $40 \%$, e esterco bovino mais casca de arroz moída tratada com hidróxido de sódio a 10 e $40 \%$. Conclui-se que a adição de casca de arroz ao esterco bovino favorece a multiplicação e o desenvolvimento de E. fetida.
\end{abstract}

Termos para indexação: Minhocas, multiplicação, substrato, Eisenia fetida.

\section{ABSTRACT}

The use of earthworms for animal feeding has been adopted and studied, because these organisms are rich in vitamins and essential amino acids. Among the organic materials that could be used in the earthworm breeding, highly available agroindustrial residues such as rice husk are being currently used in Rio Grande do Sul. The aim of this work was to evaluate substrate based on rice husk and cattle manure on the multiplication and biomass of earthworms (Eisenia fetida). In this work, the population of earthworms, the cocoon numbers, the multiplication index, as well as fresh and dry biomass of earthworms were evaluated. The evaluation was performed 60 days after the installation of the experiment and each treatment had four replications. The statistical analysis of the results showed that the inclusion of rice husk favored the development and rate of earthworm reproduction. The highest earthworm biomass was obtained in the treatments $50 \%$ rice husk and $50 \%$ cattle manure, cattle manure plus rice husk with ammonium hydroxide at 20 and $40 \%$, and cattle bovine plus ground rice husk with sodium hydroxide at 10 and $40 \%$. It was concluded that the addition of rice husk to the cattle manure was efficient in the multiplication and development of E. fetida.

Index terms: Earthworms, multiplication, substrate, Eisenia fetida.

(Recebido em 13 de setembro de 2007 e aprovado em 4 de junho de 2008)

\section{INTRODUÇÃO}

As minhocas apresentam um elevado potencial na transformação de resíduos orgânicos. Esses organismos são utilizados há milênios na alimentação humana por algumas populações do continente africano. Os chineses, há mais de 2000 anos, consomem minhocas desidratadas como uma fonte protéica alternativa. Na natureza, por ser uma presa fácil e rica em nutrientes, a minhoca constitui parte da alimentação de um grande número de animais, como peixes, aves, répteis e mamíferos (ANTONIOLLI et al., 1996; LEE, 1985).

Nos últimos anos, a produção de minhocas tem se popularizado (KHATOUNAIN, 2001) e a atividade tem atraído grande interesse em função da utilização da biomassa de minhocas como proteína, na alimentação animal e iscas. Além disso, tem sido utilizada na produção de húmus, fertilizante orgânico útil na produção vegetal (EDWARDS, 2004). Com o desenvolvimento da minhocultura nacional, alguns pesquisadores estão

'Bióloga, Doutora em Ciência do Solo - Departamento de Solos - Universidade Federal de Santa Maria/UFSM - Avenida Roraima, 1000 - Camobi 97105-900 - Santa Maria, RS - zantoniolli@gmail.com

Engenheira Agrônoma, Doutoranda do Programa de Pós-graduação em Ciência do Solo - Departamento de Solos - Universidade Federal de Santa Maria/ UFSM - Avenida Roraima, 1000 - Camobi - 97105-900 - Santa Maria, RS - ge.pauli@yahoo.com.br

${ }^{3}$ Engenheiro Agrônomo, Doutorando do Programa de Pós-graduação em Ciência do Solo - Departamento de Solos - Universidade Federal de Santa Maria UFSM - Avenida Roraima, 1000 - Camobi - 97105-900 - Santa Maria, RS - bemfica_steffen@yahoo.com.br

Ciênc. agrotec., Lavras, v. 33, n. 3, p. 824-830, maio/jun., 2009 
desenvolvendo técnicas com o objetivo de buscar um substrato que favoreça a sua multiplicação e aumento na biomassa, visando a sua transformação em farinha ou concentrado protéico.

A farinha de minhoca incluída nas dietas de aves e suínos pode promover resultados superiores às fontes protéicas de origem animal tradicionalmente utilizadas (SABINE, 1983). Dessa forma, o interesse na transformação da biomassa de minhocas em um complemento alimentar eficiente para animais incentivou a atividade da minhocultura no país, inclusive as pesquisas na busca de substratos e espécies de minhocas com maiores aptidões para a atividade.

A espécie E. fétida (Savigny, 1826), também conhecida como vermelha da Califórnia, vem sendo amplamente utilizada na minhocultura. De acordo com Aquino \& Nogueira (2001) e Dutra (2001), isso se deve à alta capacidade de proliferação, ao crescimento rápido e à elevada resistência apresentada por essa espécie, além da alta capacidade para transformar os mais diversos tipos de resíduos orgânicos (ATIYEH et al., 2000).

No Brasil, o principal material orgânico utilizado como matéria prima para a criação de minhocas e produção de húmus é o esterco bovino. No entanto, existem diversos outros materiais que podem ser utilizados (EDWARDS, 2004), como os resíduos orgânicos oriundos da atividade agrícola (DUTRA, 2001; SILVA et al., 2002). Morselli et al. (1997) utilizaram casca de arroz, um resíduo agrícola de elevada disponibilidade, mas reduzido aproveitamento no estado do Rio Grande do Sul. Devido ao fato de, aproximadamente, $60 \%$ dos resíduos ingeridos pelas minhocas serem transformados em vermicomposto (ANTONIOLLI et al., 2002), a utilização de casca de arroz na minhocultura poderia trazer benefícios tanto para os criadores de minhocas, quanto para os engenhos de arroz e para o ambiente.

A sociedade moderna, com sua alta densidade populacional, sofisticadas indústrias e métodos intensivos de agricultura, produz cada vez mais, uma quantidade crescente de resíduos. Entre os resíduos produzidos, principalmente na área de beneficiamento, está a casca do arroz, que representa $20 \%$ do peso total da produção (FOLETTO et al., 2005). Segundo dados do IBGE (2006) sobre a safra de 2005, o Brasil produziu 13 milhões de toneladas de arroz. No estado do Rio Grande do Sul, na safra 2005/06, foi plantado um milhão de hectares, gerando uma produção de 6.886.091 toneladas de arroz (IRGA, 2005), a qual, após o beneficiamento é capaz de gerar, aproximadamente, 1.377.218 toneladas de casca (AZAMBUJA, 2004). A quase totalidade desse resíduo acaba sendo rejeitada pelos orizicultores, depositado nas proximidades dos engenhos, descartado em lavouras e fundos de rios ou incinerado em ambientes fechados, cujos processos de combustão e gaseificação formam partículas de cinzas tóxicas e prejudiciais ao homem (FOLETTO et al., 2005; PEROZZI, 2004; SOUZA, 1993).

O descarte de resíduos orgânicos é uma problemática crescente que vem sendo enfrentada por muitos países, mas que poderia ser amenizada por meio da adoção de práticas simples, como a minhocultura e a vermicompostagem. No entanto, a participação desses organismos no processo restringe-se a projetos de pesquisas em instituições de ensino.

Este trabalho buscou avaliar a eficiência de substratos constituídos à base de casca de arroz submetida a tratamentos físicos (moagem e carbonização) e químico (utilização de diferentes álcalis) e esterco bovino na multiplicação e produção de biomassa de minhocas frescas e secas, visando maior produção de biomassa.

\section{MATERIAL E MÉTODOS}

O trabalho foi realizado em casa de vegetação, nos meses de julho a setembro de 2006. Os materiais utilizados como substrato para o experimento foram: esterco curtido de bovinos, casca de arroz moída e carbonizada (tratamentos físicos), casca de arroz inteira, casca de arroz tratada quimicamente com quatro álcalis: hidróxidos de cálcio $\left(\mathrm{Ca}(\mathrm{OH})_{2}\right)$, potássio $(\mathrm{KOH})$, amônio $\left(\mathrm{NH}_{4} \mathrm{OH}\right)$ e sódio $(\mathrm{NaOH})$. Os tratamentos foram definidos pela combinação de dois métodos de tratamento: um físico (carbonização e moagem da casca de arroz em moinho marca Marconi, modelo MA 340) e um químico (tratamento com álcalis), o qual foi realizado com o objetivo de acelerar a decomposição da casca de arroz (MARTINEZ, 1981). O tratamento controle foi tratado com a correspondente quantidade de água (álcali $0 \%$ ). As minhocas utilizadas foram E. fetida adultas (cliteladas).

Para o tratamento químico da casca de arroz, utilizouse o método úmido ou por imersão (MARTINEZ, 1981), sendo utilizados quatro níveis de concentração para cada álcali: 0 (controle), 10, 20 e $40 \mathrm{~g}$ de álcali $100 \mathrm{~g}^{-1}$ de matéria seca de casca de arroz. Utilizou-se volume total de quatro litros de substrato para cada tratamento.

As unidades experimentais constaram de sacos plásticos pretos com capacidade para oito litros, nos quais foram adicionados o substrato correspondente a cada tratamento e seis minhocas adultas da espécie E. fetida. Essa quantidade de organismos colocados no substrato foi designada de modo a oferecer condições adequadas para o desenvolvimento das minhocas. 
As avaliações foram realizadas 60 dias após a instalação do experimento, sendo avaliados o número de indivíduos jovens, adultos e cocons, a massa de minhocas frescas e secas e o índice de multiplicação das minhocas (população final / população inicial).

A população de minhocas foi obtida por meio de contagem manual. Todo o material de cada unidade experimental foi colocado sobre um plástico branco onde foram separadas as minhocas jovens, adultas e os cocons do vermicomposto. Os indivíduos coletados em cada unidade experimental foram colocados em frascos com água limpa, onde permaneceram durante 24 horas para que todo o material presente em seu trato digestivo fosse excretado (GIRACCA, 2005). Posteriormente, foram retiradas da água, secas em papel toalha e pesadas para a obtenção da massa de minhocas frescas. Após a pesagem, as minhocas foram mantidas 72 horas em estufa a $60^{\circ} \mathrm{C}$, em recipientes abertos forrados com papel alumínio, para a obtenção de peso constante. As amostras foram retiradas da estufa e pesadas para a obtenção da massa de minhocas secas.

O delineamento experimental utilizado foi o inteiramente casualizado, com quatro repetições. O número de minhocas jovens, adultas e cocons, bem como a massa de minhocas frescas e secas foram transformados para raiz quadrada de $\mathrm{x}+0,5$ e submetidos à análise de variância e teste de médias pelo teste de Scott-Knott, a 5\% de probabilidade, utilizando o programa estatístico SISVAR (FERREIRA, 2000).

\section{RESULTADOS E DISCUSSÃO}

Analisando-se os resultados referentes ao número final de minhocas jovens, observa-se que não houve diferença estatística significativa entre os tratamentos constituídos por $50 \%$ de casca de arroz inteira (CAI), 50\% de casca de arroz moída (CAM) e 50\% de casca de arroz carbonizada, casca de arroz inteira, tratada com $\mathrm{NH}_{4} \mathrm{OH}$ em todas as concentrações utilizadas, $\mathrm{Ca}(\mathrm{OH})_{2}$ a $40 \%$ e casca de arroz moída tratada com $\mathrm{NH}_{4} \mathrm{OH}$ a 10 e $40 \%, \mathrm{NaOH}$ a 10 e $40 \%$ e tratada com $\mathrm{Ca}(\mathrm{OH})_{2}$ a $40 \%$. No entanto, o maior número de organismos jovens foi verificado no tratamento casca de arroz inteira, tratada com $\mathrm{NH}_{4} \mathrm{OH} 20 \%$, obtendose o total de 187 indivíduos.

O maior número de cocons foi obtido no tratamento constituído por $50 \%$ de casca de arroz carbonizada, no qual foram encontrados 132 cocons a partir da inoculação de apenas seis matrizes (Tabela 1). Embora esse tratamento tenha diferido estatisticamente dos demais quanto ao número total de cocons, não diferiu significativamente do tratamento constituído por casca de arroz inteira (50\%) e esterco bovino (50\%) quanto ao número de minhocas jovens, adultas e índice de multiplicação.

Quanto ao índice de multiplicação, parâmetro que avalia a capacidade reprodutiva das matrizes em determinado ambiente, o tratamento casca de arroz inteira (50\%) tratada com $\mathrm{NH}_{4} \mathrm{OH}$ a $20 \%$ apresentou o melhor resultado $(32,33)$, seguido dos tratamentos casca de arroz inteira (50\%), casca de arroz moída (50\%) tratada com $\mathrm{NH}_{4} \mathrm{OH}$ a $10 \%$ e casca de arroz moída tratada com $\mathrm{NaOH}$ a $20 \%$, no entanto, sem apresentar diferenças estatisticamente significativas.

Os tratamentos que proporcionaram menor índice de multiplicação de minhocas foram casca de arroz inteira (100\%), casca de arroz moída (100\%), casca de arroz moída tratada com $\mathrm{KOH}$ a $40 \%$ e com $\mathrm{NH}_{4} \mathrm{OH}$ a $20 \%$. Nos tratamentos onde houve a utilização apenas da casca de arroz moída, o valor médio final de minhocas encontradas foi inferior ao número de matrizes inoculadas, resultando em índice de multiplicação igual a zero (Tabela 1).

O tratamento com esterco bovino (100\%), embora tenha sido observado índice de multiplicação de 8,5, apresentou número de minhocas adultas, jovens e cocons superior aos apresentados por Aquino et al. (1994), os quais obtiveram índice de multiplicação 4,54 e apenas 4, 18,5 e 1,5 indivíduos adultos, jovens e cocons, respectivamente, 60 dias após a inoculação de 5 matrizes de E. fetida. Pereira et al. (2005) encontraram índice de multiplicação de 2,09 para minhocas inoculadas em esterco bovino (100\%), ao final de 62 dias.

$\mathrm{O}$ tratamento da casca de arroz com $\mathrm{NH}_{4} \mathrm{OH}$ favoreceu uma alta multiplicação das minhocas, resultando no maior número de jovens (187) e adultos (9) encontrados nas unidades experimentais (Tabela 1). Provavelmente, os melhores resultados observados quando se adicionou $\mathrm{NH}_{4} \mathrm{OH}$ ao substrato estejam relacionados à adição de nitrogênio ao meio, o qual é fundamental para a manutenção da microbiota presente no trato digestivo das minhocas, atuando na transformação dos resíduos orgânicos.

Nas avaliações referentes à biomassa de minhocas, os tratamentos casca de arroz inteira, tratadas com $\mathrm{NH}_{4} \mathrm{OH}$ a 20 e $40 \%$, casca de arroz moída tratadas com $\mathrm{NaOH}$ a $10 \%$ e $50 \%$ casca de arroz inteira mais $50 \%$ esterco bovino proporcionaram maior biomassa de minhocas frescas. Quanto à biomassa de minhocas secas, verificou-se que os tratamentos que apresentaram os melhores resultados foram os mesmos que apresentaram os maiores valores para a biomassa de minhocas frescas, além dos tratamentos casca de arroz inteira tratadas com $\mathrm{NH}_{4} \mathrm{OH}, \mathrm{Ca}(\mathrm{OH})_{2}$ e $\mathrm{NaOH}$ a 10, 40 e $40 \%$ respectivamente, e casca de arroz moída tratada com $\mathrm{NaOH}$ a $40 \%$ (Tabela 1). 
Tabela 1 - Número de minhocas jovens e adultas, número de cocons e índice de multiplicação obtidos nos tratamentos à base de esterco curtido bovino (EB), casca de arroz carbonizada, casca de arroz inteira (CAI) e casca de arroz moída (CAM), tratadas com diferentes porcentuais de $\mathrm{NH}_{4} \mathrm{OH}, \mathrm{NaOH}, \mathrm{Ca}(\mathrm{OH})_{2}$ e $\mathrm{KOH}$. Média de quatro repetições.

\begin{tabular}{|c|c|c|c|c|c|c|}
\hline \multirow[t]{2}{*}{ Tratamento } & \multicolumn{2}{|c|}{$\mathrm{N}^{\mathrm{o}}$ de minhocas } & \multirow{2}{*}{$\begin{array}{l}\mathrm{N}^{\mathrm{o}} \text { de } \\
\text { cocons }\end{array}$} & \multirow[t]{2}{*}{$\mathrm{IM}^{* *}$} & \multicolumn{2}{|c|}{$\begin{array}{l}\text { Biomassa de } \\
\text { minhocas }(\mathrm{g})\end{array}$} \\
\hline & Jovens & Adultas & & & Frescas & Secas \\
\hline CAI (100\%) & $0 \mathrm{~d}^{*}$ & $0 \mathrm{c}$ & $0 \mathrm{~d}$ & $0,00 \mathrm{c}$ & $0,00 \mathrm{e}$ & $0,00 \mathrm{~d}$ \\
\hline $\mathrm{CAI}(50 \%)+\mathrm{EB}(50 \%)$ & $173 \mathrm{a}$ & $6 \mathrm{a}$ & $73 \mathrm{~b}$ & $29,66 \mathrm{a}$ & $18,78 \mathrm{a}$ & $2,50 \mathrm{a}$ \\
\hline $\mathrm{CAI}\left(\mathrm{NH}_{4} \mathrm{OH} 10 \%\right)+\mathrm{EB}(50 \%)$ & $130 \mathrm{a}$ & 9 a & $77 \mathrm{~b}$ & $23,16 \mathrm{a}$ & $17,62 \mathrm{~b}$ & $2,41 \mathrm{a}$ \\
\hline $\mathrm{CAI}\left(\mathrm{NH}_{4} \mathrm{OH} 20 \%\right)+\mathrm{EB}(50 \%)$ & $187 \mathrm{a}$ & $7 \mathrm{a}$ & $92 \mathrm{~b}$ & $32,33 \mathrm{a}$ & $20,35 \mathrm{a}$ & $2,53 \mathrm{a}$ \\
\hline $\mathrm{CAI}\left(\mathrm{NH}_{4} \mathrm{OH} 40 \%\right)+\mathrm{EB}(50 \%)$ & $144 \mathrm{a}$ & $5 \mathrm{a}$ & $67 \mathrm{~b}$ & $24,83 \mathrm{a}$ & $19,71 \mathrm{a}$ & $2,12 \mathrm{a}$ \\
\hline $\mathrm{CAI}(\mathrm{NaOH} 10 \%)+\mathrm{EB}(50 \%)$ & $70 \mathrm{~b}$ & $4 \mathrm{~b}$ & $41 \mathrm{c}$ & $12,33 \mathrm{~b}$ & $11,09 \mathrm{c}$ & $1,24 \mathrm{c}$ \\
\hline $\mathrm{CAI}(\mathrm{NaOH} 20 \%)+\mathrm{EB}(50 \%)$ & $76 \mathrm{~b}$ & $7 \mathrm{a}$ & $37 \mathrm{c}$ & $14,00 \mathrm{~b}$ & $14,82 \mathrm{c}$ & $1,74 \mathrm{~b}$ \\
\hline $\mathrm{CAI}(\mathrm{NaOH} 40 \%)+\mathrm{EB}(50 \%)$ & $88 \mathrm{~b}$ & 8 a & $72 \mathrm{~b}$ & $16,00 \mathrm{~b}$ & $15,52 \mathrm{~b}$ & $2,05 \mathrm{a}$ \\
\hline $\mathrm{CAI}\left(\mathrm{Ca}(\mathrm{OH})_{2} 10 \%\right)+\mathrm{EB}(50 \%)$ & $99 \mathrm{~b}$ & $7 \mathrm{a}$ & $48 \mathrm{c}$ & $17,66 \mathrm{~b}$ & $12,49 \mathrm{c}$ & $1,69 \mathrm{~b}$ \\
\hline $\mathrm{CAI}\left(\mathrm{Ca}(\mathrm{OH})_{2} 20 \%\right)+\mathrm{EB}(50 \%)$ & $88 \mathrm{~b}$ & $6 \mathrm{a}$ & $55 \mathrm{~b}$ & $15,83 \mathrm{~b}$ & $12,73 \mathrm{c}$ & $1,69 \mathrm{~b}$ \\
\hline $\mathrm{CAI}\left(\mathrm{Ca}(\mathrm{OH})_{2} 40 \%\right)+\mathrm{EB}(50 \%)$ & $114 \mathrm{a}$ & $8 \mathrm{a}$ & $68 \mathrm{~b}$ & $20,33 \mathrm{a}$ & $15,86 \mathrm{~b}$ & $2,08 \mathrm{a}$ \\
\hline $\mathrm{CAI}(\mathrm{KOH} 10 \%)+\mathrm{EB}(50 \%)$ & $74 \mathrm{~b}$ & $5 \mathrm{a}$ & $41 \mathrm{c}$ & $13,33 \mathrm{~b}$ & $10,59 \mathrm{c}$ & $1,34 \mathrm{c}$ \\
\hline CAI (KOH 20\%) + EB (50\%) & $80 \mathrm{~b}$ & $6 \mathrm{a}$ & $39 \mathrm{c}$ & $14,33 \mathrm{~b}$ & $12,56 \mathrm{c}$ & $1,68 \mathrm{~b}$ \\
\hline $\mathrm{CAI}(\mathrm{KOH} 40 \%)+\mathrm{EB}(50 \%)$ & $79 \mathrm{~b}$ & $7 \mathrm{a}$ & $59 \mathrm{~b}$ & $14,33 \mathrm{~b}$ & $12,06 \mathrm{c}$ & $1,56 \mathrm{~b}$ \\
\hline CAM (100\%) & $1 \mathrm{~d}$ & $3 \mathrm{~b}$ & $2 \mathrm{~d}$ & $0,00 \mathrm{c}$ & $1,38 \mathrm{e}$ & $0,17 \mathrm{~d}$ \\
\hline $\operatorname{CAM}(50 \%)+\mathrm{EB}(50 \%)$ & $136 \mathrm{a}$ & $6 \mathrm{a}$ & $52 \mathrm{c}$ & $23,66 \mathrm{a}$ & $11,75 \mathrm{c}$ & $1,48 \mathrm{~b}$ \\
\hline $\mathrm{CAM}\left(\mathrm{NH}_{4} \mathrm{OH} 10 \%\right)+\mathrm{EB}(50 \%)$ & $169 \mathrm{a}$ & $6 \mathrm{a}$ & $74 \mathrm{~b}$ & $29,33 \mathrm{a}$ & $14,68 \mathrm{c}$ & $1,75 \mathrm{~b}$ \\
\hline $\mathrm{CAM}\left(\mathrm{NH}_{4} \mathrm{OH} 20 \%\right)+\mathrm{EB}(50 \%)$ & $44 \mathrm{c}$ & $1 \mathrm{c}$ & $19 \mathrm{~d}$ & $7,50 \mathrm{c}$ & $3,41 \mathrm{e}$ & $0,47 \mathrm{~d}$ \\
\hline $\mathrm{CAM}\left(\mathrm{NH}_{4} \mathrm{OH} 40 \%\right)+\mathrm{EB}(50 \%)$ & $148 \mathrm{a}$ & $5 \mathrm{a}$ & $60 \mathrm{~b}$ & $25,50 \mathrm{a}$ & $13,19 \mathrm{c}$ & $1,71 \mathrm{~b}$ \\
\hline CAM (NaOH 10\%) + EB (50\%) & $162 \mathrm{a}$ & $6 \mathrm{a}$ & $47 \mathrm{c}$ & $28,16 \mathrm{a}$ & $20,26 \mathrm{a}$ & $2,19 \mathrm{a}$ \\
\hline $\mathrm{CAM}(\mathrm{NaOH} 20 \%)+\mathrm{EB}(50 \%)$ & $65 \mathrm{~b}$ & $6 \mathrm{a}$ & $25 \mathrm{~d}$ & $12,00 \mathrm{~b}$ & $15,38 \mathrm{~b}$ & $1,70 \mathrm{~b}$ \\
\hline $\mathrm{CAM}(\mathrm{NaOH} 40 \%)+\mathrm{EB}(50 \%)$ & $147 \mathrm{a}$ & $6 \mathrm{a}$ & $81 \mathrm{~b}$ & $25,50 \mathrm{a}$ & $15,86 \mathrm{~b}$ & $2,06 \mathrm{a}$ \\
\hline $\mathrm{CAM}\left(\mathrm{Ca}(\mathrm{OH})_{2} 10 \%\right)+\mathrm{EB}(50 \%)$ & $103 \mathrm{~b}$ & $6 \mathrm{a}$ & $66 \mathrm{~b}$ & $18,16 \mathrm{~b}$ & $9,46 \mathrm{~d}$ & $1,23 \mathrm{c}$ \\
\hline $\mathrm{CAM}\left(\mathrm{Ca}(\mathrm{OH})_{2} 20 \%\right)+\mathrm{EB}(50 \%)$ & $85 \mathrm{~b}$ & $4 \mathrm{~b}$ & $40 \mathrm{c}$ & $15,00 \mathrm{~b}$ & $7,21 \mathrm{~d}$ & $0,92 \mathrm{c}$ \\
\hline $\mathrm{CAM}\left(\mathrm{Ca}(\mathrm{OH})_{2} 40 \%\right)+\mathrm{EB}(50 \%)$ & $136 \mathrm{a}$ & $6 \mathrm{a}$ & $80 \mathrm{~b}$ & $23,66 \mathrm{a}$ & $10,55 \mathrm{c}$ & $1,44 \mathrm{~b}$ \\
\hline CAM (KOH 10\%) + EB (50\%) & $83 \mathrm{~b}$ & $7 \mathrm{a}$ & $67 \mathrm{~b}$ & $15,00 \mathrm{~b}$ & $10,19 \mathrm{c}$ & $1,30 \mathrm{c}$ \\
\hline CAM (KOH $20 \%)+\mathrm{EB}(50 \%)$ & $92 \mathrm{~b}$ & $6 \mathrm{a}$ & $87 \mathrm{~b}$ & $16,33 \mathrm{~b}$ & $11,28 \mathrm{c}$ & $1,52 \mathrm{~b}$ \\
\hline $\mathrm{CAM}(\mathrm{KOH} 40 \%)+\mathrm{EB}(50 \%)$ & $47 \mathrm{c}$ & $3 \mathrm{~b}$ & $60 \mathrm{~b}$ & $8,50 \mathrm{c}$ & $6,73 \mathrm{~d}$ & $0,86 \mathrm{c}$ \\
\hline $\begin{array}{l}\text { Casca de arroz carbonizada }(50 \%) \\
+ \text { Esterco bovino }(50 \%)\end{array}$ & $159 \mathrm{a}$ & $6 \mathrm{a}$ & $132 \mathrm{a}$ & 27,66 a & $14,03 \mathrm{c}$ & $1,70 \mathrm{~b}$ \\
\hline Esterco bovino $(100 \%)$ & $44 \mathrm{c}$ & $6 \mathrm{a}$ & $57 \mathrm{~b}$ & $8,50 \mathrm{c}$ & $8,56 \mathrm{~d}$ & $1,20 \mathrm{c}$ \\
\hline $\mathrm{CV} * * *(\%)$ & 15,90 & 6,27 & 14,23 & 14,74 & 5,41 & 7,61 \\
\hline
\end{tabular}

* Médias seguidas da mesma letra nas colunas não diferem entre si pelo teste de Scott-Knott, a 5\% de probabilidade.

** (IM) Índice de multiplicação (População final / População inicial).

*** Coeficiente de variação 
Os tratamentos constituídos por $100 \%$ casca de arroz inteira, $100 \%$ casca de arroz moída e $50 \%$ casca de arroz moída, tratada com $\mathrm{NH}_{4} \mathrm{OH}$ a $20 \%$ foram os que apresentaram os menores valores de biomassa de minhocas frescas e secas (Tabela 1). A não permanência ou morte das matrizes inoculadas nos tratamentos casca de arroz inteira (100\%) e casca de arroz moída (100\%) foi provocada, provavelmente, pela falta de alimento oferecido às minhocas nesses substratos.

No tratamento esterco bovino (100\%), substrato comumente utilizado pelos minhocultores, verificou-se a presença de 44 minhocas jovens e 57 cocons. Esses valores foram inferiores aos encontrados no tratamento onde houve adição de $50 \%$ de casca de arroz inteira ao esterco bovino (Tabela 1). Os resultados desse trabalho sugerem que a inclusão de casca de arroz favoreceu o desenvolvimento e a taxa de reprodução das minhocas, o que pode ter sido influenciado pela textura, umidade e aeração do substrato, devido à mistura de casca de arroz ao esterco bovino. Segundo Migdalski (2001), a mistura de resíduos vegetais fibrosos ao esterco é uma alternativa eficiente para evitar a compactação e consequente redução da aeração e drenagem do esterco, o que tende a ocorrer naturalmente com o passar do tempo, dificultando a movimentação e alimentação das minhocas (MARTINEZ, 1998).

Trabalhos realizados por Morselli \& Valente (1997), avaliando o desenvolvimento e a capacidade reprodutiva de E. fetida em diferentes substratos formados a partir da associação de várias matérias-primas como esterco, casca de arroz, bagaço de laranja e verduras, mostraram que esses indivíduos apresentam um melhor desenvolvimento, quando colocados em material misturado com casca de arroz. Segundo os autores, isso se deve, provavelmente, ao maior teor de sílica presente, uma vez que as minhocas necessitam desse mineral, além da casca de arroz favorecer a aeração do substrato. Schiavon et al. (2007), avaliando os efeitos da adição de diferentes proporções de casca de arroz natural ( 25 e $50 \%$ ) e casca de arroz carbonizada (25 e $50 \%$ ) ao esterco bovino, na multiplicação e reprodução de E. fetida, observaram que a adição de $25 \%$ de casca de arroz natural ao esterco proporcionou as melhores condições para o desenvolvimento das minhocas, favorecendo a sua locomoção e respiração.

Embora a utilização do álcali $\mathrm{NH}_{4} \mathrm{OH}$, a $20 \%$ no tratamento de casca de arroz inteira, tenha proporcionado a maior multiplicação das minhocas, quando utilizado no tratamento da casca de arroz moída proporcionou os menores índices de multiplicação. Provavelmente, esse comportamento seja resultante de um ambiente não favorável ao desenvolvimento da minhoca, devido a menor aeração e maior compactação do substrato quando da utilização da casca de arroz moída, a qual, quando úmida, tende a formar uma massa densa. Segundo Aquino et al. (1994) a maturidade sexual das minhocas está relacionada com as condições do meio em que se encontram, resultando em uma maior ou menor multiplicação em determinado período de tempo.

A atividade e a maturação sexual das minhocas são influenciadas pelas condições do meio em que vivem. Nas condições em que foi realizado este trabalho, verificou-se que alguns indivíduos atingiram sua maturidade sexual em menos de 60 dias. Esses resultados estão de acordo com Aquino et al. (2001) e Domínguez \& Edwards (1997), os quais afirmam que, em condições favoráveis, as minhocasfilhas da espécie E. fetida atingem a maturidade sexual com completa formação do clitelo, dentro de 40 a 60 dias após a eclosão. Segundo Antoniolli et al. (2002), a atividade biológica das minhocas diminui em função das baixas temperaturas.

\section{CONCLUSÕES}

A adição de casca de arroz ao esterco bovino favoreceu a multiplicação e o desenvolvimento da Eisenia fetida.

A utilização de casca de arroz inteira, casca de arroz moída e casca de arroz carbonizada $(50 \%)$, casca de arroz inteira tratada com $\mathrm{NH}_{4} \mathrm{OH}$ a 10,20 e $40 \%, \mathrm{Ca}(\mathrm{OH})_{2}$ a $40 \%$ e casca de arroz moída tratada com $\mathrm{NH}_{4} \mathrm{OH}$ a 10 e $40 \%$, $\mathrm{NaOH}$ a 10 e $40 \%$ e tratada com $\mathrm{Ca}(\mathrm{OH})_{2}$ a $40 \%$ proporcionou maior desenvolvimento de organismos jovens, adultos e maior multiplicação das minhocas.

A utilização de casca de arroz carbonizada juntamente com esterco curtido de bovino favoreceu a produção de cocons pelas minhocas.

A moagem da casca de arroz, bem como seu tratamento com álcalis favoreceu a produção de biomassa de E. fetida.

\section{AGRADECIMENTOS}

Ao apoio financeiro disponibilizado pela FIEX/ UFSM, Departamento de Solos, ao CNPq pela Bolsa de Produtividade e à CAPES pelas Bolsas de Doutorado.

\section{REFERÊNCIAS BIBLIOGRÁFICAS}

ANTONIOLLI, Z. I.; GIRACCA, E. M. N.; BARCELLOS, L. A.; VENTURINI, S. F.; VENTURINI, E. F.; WIETHAN, M. M. S.; CARLOSSO, S. J. T.; BENEDETTI, T.; SENHOR, T. C.; SANTI, G. R. Minhocultura e vermicompostagem. Santa Maria: UFSM, 2002. 24 p. (Boletim técnico, 3). 
ANTONIOLLI, Z. I.; GIRACCA, E. M. N.; CARLOSSO, S. J. T.; WIETHAN, M. M. S.; FERRI, M. Iniciação à minhocultura. Santa Maria: UFSM, 1996. 96 p.

AQUINO, A. M. de; ALMEIDA, D. L. de; FREIRE, L. R.; DE-POLLI, H. Reprodução de minhocas (Oligochaeta) em esterco bovino e bagaço de cana-deaçúcar. Pesquisa Agropecuária Brasileira, Brasília, v. 29, n. 2, p. 161-168, 1994.

AQUINO, A. M.; NOGUEIRA, E. M. Fatores limitantes da vermicompostagem de esterco suíno e de aves e influência da densidade populacional das minhocas na sua reprodução. Seropédica: Embrapa Agrobiologia, 2001. 10 p. (Documentos, 147).

ATIYEH, R. M.; DOMÍNGUEZ, J.; SUBLER, S.; EDWARDS, C. A. Changes in biochemical properties of cow manure during processing by earthworms (Eisenia andrei, Bouché) and the effects on seedling growth. Pedobiologia, Jena, v. 44, n. 6, p. 709-724, 2000.

AZAMBUJA, I. H. V. Aspectos socioeconômicos da produção de arroz. In: GOMES, A. S. da. Arroz irrigado no Sul do Brasil. Brasília, DF: Embrapa, 2004. p. 23-44.

DOMÍNGUEZ, J.; EDWARDS, C. A. Effects of stocking rate and moisture content on the growth and maturation of Eisenia Andrei (Oligochaeta) in pig manure. Soil Biology and Biochemistry, v. 29, n. 3/4, p. 743-746, 1997.

DUTRA, L. C. Compostagem e vermicompostagem em bagaço de uvas. 2001. 117 f. Dissertação (Mestrado em Tecnologia e Ciência dos Alimentos) - Universidade Federal de Santa Maria, Santa Maria, 2001.

EDWARDS, C. A. The use of earthworms in the breakdown and management of organic wastes. In: EDWARDS, C. A. (Org.). Earthworm ecology. Boca Raton: Saint Lucie, 2004. p. 327-354.

FERREIRA, D. F. Sistemas de análise estatística para dados balanceados. Lavras: UFLA/DEX/SISVAR, 2000. $145 \mathrm{p}$.

FOLETTO, E. L.; HOFFMANN, R.; HOFFMANN, R. S.; PORTUGAL JUNIOR, U. L.; JAHN, S. L.
Aplicabilidade das cinzas da casca de arroz. Química Nova, São Paulo, v. 28, p. 1055-1060, 2005.

GIRACCA, E. M. N. Efeito do calcário em atributos biológicos do solo. 2005. $61 \mathrm{f}$. Tese (Doutorado em Ciência do Solo) - Universidade Federal de Santa Maria, Santa Maria, 2005.

INSTITUTO BRASILEIRO DE GEOGRAFIA E ESTATÍSTICA. Levantamento sistemático da produção agrícola. Disponível em: <http:// www1ibge.gov.br/home/presidencia/noticias/ noticia-visualiza.php?.- Acesso em: 27 jun. 2006.

INSTITUTO RIOGRANDENSE DO ARROZ. Dados de safra: série histórica da área plantada, produção e rendimento. 2005. Disponível em: <http:// Www.irga.rs.gov.br/dados.htm. Acesso em: 27 jun. 2006.

KHATOUNAIN, C. A. A reconstrução ecológica da agricultura. [S.1.: s.n.], 2001. 348 p.

LEE, K. E. Earthworms: their ecology and relationships with soils and land use. Melbourne: CSIRO, 1985. $410 \mathrm{p}$.

MARTINEZ, A. A. A grande e poderosa minhoca: manual prático do minhocultor. 4. ed. Jaboticabal: Funep, 1998. 148 p.

MARTINEZ, M. S. G. Efeitos do tratamento químico com diversos álcalis sobre a composição química e digestibilidade da casca de arroz. 1981. $156 \mathrm{f}$.

Dissertação (Mestrado em Zootecnia) - Universidade Federal de Santa Maria, Santa Maria, 1981.

MIGDALSKI, M. C. Criação de minhocas. Viçosa, MG: Aprenda Fácil, 2001. 118 p.

MORSELLI, T. B. G. A.; CRUZ, L. E. C. da; POCAI, D.; $\mathrm{PICH}, \mathrm{A}$. H. Efeito de diferentes resíduos no comportamento de Eisenia foetida em estação quente: I. eclosão. Revista Científica Rural, Bagé, v. 2, p. 4549, 1997.

MORSELLI, T. B. G. A.; VALENTE, B. Variação populacional de E. foetida em diferentes misturas de resíduos orgânicos oriundos da propriedade rural.

Revista Científica Rural, Bagé, v. 2, p. 54-57, 1997. 
PEREIRA, E. W. L.; AZEVEDO, C. M. da S. B.; LIBERALINO FILHO, J.; NUNES, G. H. de S.; TORQUATO, J. E.; SIMÕES, B. R. Produção de vermicomposto em diferentes proporções de esterco bovino e palha de carnaúba. Caatinga, Mossoró, v. 18, p. 112-116, 2005.

PEROZZI, M. Arroz em foco. 2004. Disponível em: ¿http://www.arroz.agr.br/site/arrozemfoco/040305.php̀) Acesso em: 18 abr. 2006.

SABINE, J. R. Earthworm as a source of food and drugs. In: SATCHELL, J. Earthworm ecology. London:

Chapman and Hall, 1983. p. 285-296.
SCHIAVON, G. de A.; SCHIEDECK, G.; ARAÚJO, J. M. G.; SCHWENGBER, J. E. Efeito da casca de arroz no crescimento e reprodução de minhocas. Revista Brasileira de Agroecologia, Porto Alegre, v. 2, n. 2, p. 995-999, 2007.

SILVA, C. D. da; COSTA, L. M. da; MATOS, A. T. de; CECON, P. R.; SILVA, D. D. Vermicompostagem de lodo de esgoto urbano e bagaço de cana-de-açúcar. Revista Brasileira de Engenharia Agrícola Ambiental, Campina Grande, v. 6, p. 487-491, 2002.

SOUZA, F. X. de. Casca de arroz carbonizada: um substrato para propagação de plantas. Lavoura Arrozeira, Porto Alegre, v. 46, n. 406, p. 11, 1993. 